\title{
A Complete Study of the Ground State Phase Diagrams of Spin-1 Bose-Einstein Condensates in a Magnetic Field via Continuation Methods
}

\author{
Jen-Hao Chen ${ }^{\mathrm{a}}$, I-Liang Chern ${ }^{\mathrm{b}, \mathrm{c}, *}$, Weichung Wang ${ }^{\mathrm{c}}$ \\ ${ }^{a}$ Department of Applied Mathematics, National Hsinchu University of Education, Hsinchu \\ 300, Taiwan \\ ${ }^{b}$ Department of Mathematics, National Central University, Jhongli 32001, Taiwan \\ ${ }^{c}$ Institute of Applied Mathematical Sciences, National Taiwan University, Taipei 106, \\ Taiwan
}

\begin{abstract}
We present a complete investigation of the ground state patterns and phase diagrams of the spin-1 Bose-Einstein condensates (BEC) confined in a harmonic or box potential under the influence of a homogeneous magnetic field. A pseudoarclength continuation method with parameter switching technique is developed to study the BEC systems numerically. The continuation process is performed on the parameter space consisting of the spin-independent interaction, spinexchange interaction and the quadratic Zeeman (QZ) energy parameters. In the first stage of the parameter switching process, we fix the QZ energy term to be zero and vary the interaction parameters from zero to the desired physical values. Next, we fix the interaction parameters and vary the QZ energy parameter in both positive and negative regions. Two types of phase transitions are found, as we vary the QZ parameter. The first type is a transition from a two-component $(2 \mathrm{C})$ state to a three-component $(3 \mathrm{C})$ state. The second type is a symmetry breaking in the $3 \mathrm{C}$ state. Then, a phase separation of the spin components follows. In the semi-classical regime, we find that these two phase transition curves are gradually merged.
\end{abstract}

Keywords: Spin-1 Bose-Einstein condensate, continuation method, ground state, quadratic Zeeman effect, symmetry breaking, phase transition, phase separation, phase diagram.

\section{Introduction}

Bose-Einstein condensates (BEC) with spin degree of freedom, called spinor BECs, have been achieved experimentally and attracted considerable interest

\footnotetext{
* Corresponding author

Email addresses: jhchen@mail.nhcue.edu.tw (Jen-Hao Chen), chern@math.ntu.edu.tw (I-Liang Chern), wwang@ntu.edu.tw (Weichung Wang)
} 
[1, 2, 3, 4, 5] recently. By trapping all Zeeman states of an atomic species in an optical trap, these spinor BEC possess a wealth of phenomena not found in single-component condensates, including spin vortex [6], spin textures [7, 8], domain wall structure [9, 10], and spontaneous demagnetization dynamics [1], 12]. It is well known that the sign of atomic spin-exchange interaction determines the ground state property of spin-1 condensate, and leads to different quantum phases 3 3, 13, 14]. Moreover, under an applied external magnetic field, the spontaneous symmetry breaking in a ${ }^{87} \mathrm{Rb}$ spinor BEC has recently been observed 7]. The symmetry breaking phase transition in the ground state of the spin-1 antiferromagnetic BEC was also experimentally studied 15]. These experiments have opened up intriguing possibilities for exploring the ground state structure of spin-1 BEC under the effect between the spin-dependent interaction and the external magnetic field.

In the presence of an external magnetic field, the spin-1 BEC is subjected to the Zeeman effect [1, 16, 17, 18]. While the linear Zeeman (LZ) term has no influence on the system due to conservation of the magnetization [17], the ground state and its phase diagram of the spin-1 BEC in a magnetic field are profoundly affected by the quadratic Zeeman (QZ) term which describes the Zeeman energy difference in a spin-flip collision [1]. The competition between the QZ effect and the spin-exchange interaction results in a rich variety of ground state patterns. Numerous studies on the spin-1 BEC under positive QZ effect have been reported, including spin-domain formation [19, 20], and phase separation [17, 18]. Recently, some experiments have been performed to observe the dynamics of the spin- 1 condensates of sodium atoms by using an off-resonant microwave field to rapidly switch QZ effect from positive to negative 21, 22]. This motivates us to numerically investigate the ground state patterns and phase transitions in whole range (positive and negative) of QZ effect.

The ground state structure of the spin- 1 condensate in the absence of an external magnetic field has been well examined both theoretically [23, 24, 25] and numerically [26, 14, 27]. The essential feature of these works is that, in ferromagnetic systems the ground state contains all three components which are constant multiples of a scalar wave function, while the ground state contains no zero-component in antiferromagnetic systems. Moreover, no bifurcation is found on the ground state solution curve as varying the spin-independent and spin-exchange interaction parameters.

For the spin-1 BEC in the presence of a homogeneous magnetic field, Lim et al. [28] proposed a numerical method based on the normalized gradient flow to compute the ground state solution of the system. They also numerically showed that for antiferromagnetic BEC in the presence of magnetic field, there exists a critical magnetization which is the transition between two- and three-component states. In [17], the authors theoretically predicted the existence of symmetry breaking phase transition of the antiferromagnetic system without the trapping potential. Matuszewski [18] also pointed out that in the case of harmonic trapping potentials an antiferromagnetic system can possess three distinct ground state patterns, while a ferromagnetic system possess two. However, the asymmetric solutions were drawn only schematically in their paper. In addition, the 
phase diagram in the negative QZ effect regime has not been studied before.

In this work, we aim to provide a complete investigation on the ground state patterns and the phase diagram of the spin-1 BECs confined in a harmonic or box trap with positive to negative QZ effect. Based on the pseudo-arclength continuation method (PACM) 29, 30, 31], we develop a numerical scheme to study these problems. First, together with the characteristics of the ground state of the spin-1 condensate without magnetic field [14], we trace the ground state solution curve varied by the spin-independent and spin-exchange coupling constants. We next treat the QZ effect as a continuation parameter, and take the target solution under zero magnetic field as the starting state to trace the ground state solution curve subject to nonzero magnetic field. We also detect the bifurcation point in the continuation process. The results reveal a rich variety phase transition phenomena in which symmetric or asymmetric ground states are observed. These ground states also show the miscibility or immiscibility in the three hyperfine components, and possess domain wall structures. Finally, we study the phase diagrams of antiferromagnetic systems in the semi-classical regime, in which the determination of the phase transitions is a demanding task due to the nearness of the bifurcation points. In the limiting case (zero kinetic energy term), the above two phase transition curves merge. To the best of our knowledge, some results have never been reported before.

The paper is organized as follows. In the next section, we introduce the model for the spin-1 BEC subject to an external magnetic field. In Section 3 we describe the numerical scheme based on the PACM to explore how the QZ effect and the spin-exchange interaction affect the ground state structures of the systems. In Section 4 we present the numerical results of the spin-1 BEC confined in the harmonic or box trap. The ferromagnetic or antiferromagnetic condensates with positive or negative QZ effects will be considered. Finally, conclusion of the paper is given in Section 5 .

\section{The Spin-1 BEC Models}

At sufficiently low temperature, spin- 1 atomic condensates in a nonzero homogeneous magnetic field are described by the following dimensionless Hamiltonian [16, 32, 18]:

$\mathcal{E}[\Psi]=\int_{\mathbb{R}^{3}}\left[\sum_{j=-1}^{1} \psi_{j}^{*}\left(\frac{-\nabla^{2}}{2}+V_{e x t}\right) \psi_{j}+\frac{1}{2} g_{n}|\Psi|^{4}+\frac{1}{2} g_{s}\left(\Psi^{*} S \Psi\right)^{2}-p \Psi^{*} S_{z} \Psi+q \Psi^{*} S_{z}^{2} \Psi\right] d r$.

Here, $\Psi(r)=\left(\psi_{1}(r), \psi_{0}(r), \psi_{-1}(r)\right)^{\top}$ is the vectorial order parameter corresponding to three hyperfine sublevels of the spin, $m_{F}=1,0,-1$, at position $r=(x, y, z)$. The $\Psi^{*}$ denotes the conjugate transpose of $\Psi$. The term $V_{e x t}$ represents the external trapping potential. The nonlinear interaction among spin- 1 condensate atoms is characterized by the spin-independent parameter $g_{n}$ and spin-exchange interaction parameter $g_{s}$. For $g_{n}<0$, the spin-independent 
interaction is attractive. For $g_{n}>0$ the spin-independent interaction is repulsive. The system is ferromagnetic if $g_{s}<0$ and antiferromagnetic if $g_{s}>0$. The terms $p$ and $q$ are LZ and QZ energy parameters, respectively. These two parameters are key factors in determining the ground state patterns of the spin-1 BECs in a magnetic field. The term $S=\left(S_{x}, S_{y}, S_{z}\right)$ is the spin-1 Pauli operator [3, 4] and

$$
S_{x}=\frac{1}{\sqrt{2}}\left(\begin{array}{ccc}
0 & 1 & 0 \\
1 & 0 & 1 \\
0 & 1 & 0
\end{array}\right), S_{y}=\frac{i}{\sqrt{2}}\left(\begin{array}{ccc}
0 & -1 & 0 \\
1 & 0 & -1 \\
0 & 1 & 0
\end{array}\right), S_{z}=\left(\begin{array}{ccc}
1 & 0 & 0 \\
0 & 0 & 0 \\
0 & 0 & -1
\end{array}\right)
$$

Due to the elastic atomic collisions characterized by $g_{n}$ and $g_{s}$, we have two conserved quantities. One is the normalization of the wave functions

$$
N=\int_{\mathbb{R}^{3}}\left(\left|\psi_{1}\right|^{2}+\left|\psi_{0}\right|^{2}+\left|\psi_{-1}\right|^{2}\right) d r=1,
$$

and the other is the total magnetization

$$
M=\int_{\mathbb{R}^{3}}\left(\left|\psi_{1}\right|^{2}-\left|\psi_{-1}\right|^{2}\right) d r,
$$

with $-1 \leq M \leq 1$. For convenience, we denote $n_{j}=\left|\psi_{j}\right|^{2}$ as the density of each spin component, $n=n_{1}+n_{0}+n_{1}$ as the total density, and $N_{j}=\int_{\mathbb{R}^{3}} n_{j} d r$ as the corresponding particle number.

By minimizing the mean field energy functional (11) subject to (3) and (4), we arrive at a set of three coupled Gross-Pitaevskii equations (CGPE)

$$
\left\{\begin{aligned}
(\mu+\lambda) \psi_{1} & =\left[\mathcal{L}+p+q+g_{s}\left(n_{1}+n_{0}-n_{-1}\right)\right] \psi_{1}+g_{s} \bar{\psi}_{-1} \psi_{0}^{2} \\
\mu \psi_{0} & =\left[\mathcal{L}+g_{s}\left(n_{1}+n_{-1}\right)\right] \psi_{0}+2 g_{s} \psi_{-1} \bar{\psi}_{0} \psi_{1} \\
(\mu-\lambda) \psi_{-1} & =\left[\mathcal{L}-p+q+g_{s}\left(n_{0}+n_{-1}-n_{1}\right)\right] \psi_{-1}+g_{s} \bar{\psi}_{1} \psi_{0}^{2}
\end{aligned}\right.
$$

where $\mathcal{L}=-\frac{\nabla^{2}}{2}+V_{\text {ext }}(r)+g_{n} n$ is the spin-independent part of the Hamiltonian. The Lagrange multipliers $\mu$ and $\lambda$ corresponding to the constraints (3) and (4) are interpreted respectively as the chemical and magnetic potentials of the spin-1 BEC.

\subsection{Settings for the numerical experiments}

In this work, we consider the particular CGPE that the LZ effect is neglected and the wave functions have constant phases. For the Zeeman effect, it is well known that a spin-1 BEC in a magnetic field is subjected to the Zeeman effect. However, the LZ effect term $p$ can be neglected due to conservation of the total magnetization in the system [7, 17, 15]. The $p$ term can be absorbed into the magnetic potentials $\lambda$. We thus investigate the ground state structures of the spin-1 BECs under a variation of the QZ effect. 
For the phases considerations, we write the wave functions $\psi_{j}=u_{j} e^{i \theta_{j}}$, where $\theta_{j}$ are constant absolute phases of the three hyperfine components. From $\left|\nabla \psi_{j}\right|^{2}=\left|\nabla u_{j}\right|^{2}+u_{j}^{2}\left|\nabla \theta_{j}\right|^{2}$, we see that a wave function with constant phase has least kinetic energy. In this case, the spin-exchange Hamiltonian becomes

$$
\frac{1}{2} g_{s}\left(\Psi^{*} S \Psi\right)^{2}=\frac{1}{2} g_{s}\left[\left(n_{1}-n_{-1}\right)^{2}+2 n_{0}\left(n_{1}+n_{-1}+2 u_{1} u_{-1} \cos \Delta \theta\right)\right],
$$

where $\Delta \theta=\theta_{1}+\theta_{-1}-2 \theta_{0}$ is called the relative phase. To achieve minimal spin-exchange interaction, $\Delta \theta$ must be taken as 0 or $\pi$. The former is called the phase-matched (PM) state, and the latter refers to the antipahse-matched (APM) state. The PM state is energetically favorable for the ferromagnetic condensate, while the APM state is for the antiferromagnetic case [16, 19, 17, 20].

Under the above considerations, the CGPE can be rewritten as

$$
\left\{\begin{aligned}
(\mu+\lambda) u_{1} & =\mathcal{L} u_{1}+q u_{1}+g_{s}\left[n_{0}\left(u_{1}-\sigma u_{-1}\right)+u_{1}\left(n_{1}-n_{-1}\right)\right] \\
\mu u_{0} & =\mathcal{L} u_{0}+g_{s} u_{0}\left(u_{1}-\sigma u_{-1}\right)^{2} \\
(\mu-\lambda) u_{-1} & =\mathcal{L} u_{-1}+q u_{-1}+g_{s}\left[n_{0}\left(u_{-1}-\sigma u_{1}\right)+u_{-1}\left(n_{-1}-n_{1}\right)\right],
\end{aligned}\right.
$$

where $\sigma=\operatorname{sign}\left(g_{s}\right)$. We note that $u_{j}$ 's are real-valued functions. It has been shown that it is positive or identical to zero 25]. Since the term $p M$ plays no role in the classification of ground states, we subtract them from the energy functional in our calculation. The corresponding reduced energy functional is

$$
\begin{aligned}
\tilde{\mathcal{E}}[\Psi]= & \int_{\mathbb{R}^{3}}\left[\sum_{j=-1}^{1} u_{j}\left(\frac{-\nabla^{2}}{2}+V_{\text {ext }}\right) u_{j}\right. \\
& +\frac{g_{n}}{2}\left(n_{1}^{2}+n_{0}^{2}+n_{-1}^{2}+2 n_{1} n_{-1}+2 n_{0}\left(n_{1}+n_{-1}\right)\right) \\
& \left.+\frac{g_{s}}{2}\left(\left(n_{1}-n_{-1}\right)^{2}+2 n_{0}\left(u_{1}-\sigma u_{-1}\right)^{2}\right)+q\left(n_{1}+n_{-1}\right)\right] d r .
\end{aligned}
$$

In short, we intend to explore the ground state patterns and phase diagrams of the spin-1 BECs in nonzero magnetic field by means of the CGPE (77) with two conservations (3) and (44). We also compute the corresponding energies via reduced energy functional (8) to confirm the validity of the ground states.

In particular, we investigate both ferromagnetic condensates ${ }^{87} \mathrm{Rb}\left(g_{n}>0\right.$ and $\left.g_{s}<0\right)$ and antiferromagnetic condensates ${ }^{23} \mathrm{Na}\left(g_{n}>0\right.$ and $\left.g_{s}>0\right)$ by assuming the toal number of the cold atoms is $10^{4}$. The spin component $u_{j}$ is regarded as vanished when the normalized particle number $N_{j}$ is less than $10^{-4}$. We assume a strong transverse trapping frequency which results in an anisotropic cigar-shaped spin- 1 condensate. That is, we assume $V_{e x t}(x)=V_{0} x^{2}$. The ground state solutions for a quasi one-dimensional (1D) condensates are thus investigated by solving the $1 \mathrm{D}$ version of (77). Here, we take $V_{0}=0.5$ for the case of the harmonic trapping potential, and $V_{0}=0$ for box trapping potential, i.e.,

$$
V_{\text {ext }}(x)= \begin{cases}0 & \text { when }-L \leq x \leq L \\ \infty & \text { otherwise }\end{cases}
$$


We then use the standard central finite difference method to approximate the model problem with the mesh size $h=0.05$ in all computations. We terminate the Newton correction in the continuation process when the relative residual in the 2 -norm is less than $10^{-12}$.

\subsection{Remarks on ground states}

First, for box trapping potential, the ground state is expected to be homogeneous away from boundaries or domain walls. They can be computed by minimizing the energy functional without the kinetic energy term. Such approximation is called the Thomas-Fermi approximation. The corresponding ground states are either a pure state or their combinations. The latter is a combination of non-overlapping pure states separated by domain walls [17, 18]. The pure states are listed below.

- A nematic state (NS) is a state in which all the atoms are in $m_{F}=0$ component, that is $\left(0, u_{0}, 0\right)$.

- A magnetized state (MS) is a state in which all the atoms are in $m_{F}=+1$ or -1 component, that is $\left(u_{1}, 0,0\right)$ or $\left(0,0, u_{-1}\right)$.

- A two-component $(2 \mathrm{C})$ state is a state in which $m_{F}= \pm 1$ components are populated and miscible. This usually happens in antiferromagnetic systems. For ferromagnetic systems, however, $m_{F}=1,0$ can be miscible. Such state $\left(u_{1}, u_{0}, 0\right)$ is called the $2 \mathrm{C}_{\{1,0\}}$ to distinguish from the previous $2 \mathrm{C}_{\{1,-1\}}$.

- A three-component (3C) state is a state in which all spin components are populated and miscible. Note that the $3 \mathrm{C}$ state is called the PM state if $\Delta \theta=0$ and the APM state if $\Delta \theta=\pi$.

Second, we summarize some features of the ground states of the spin-1 without magnetic field as follows (see [14, 25] for more details).

- For the ferromagnetic system $\left(g_{s}<0\right)$, the ground state is a constant multiple of a single wave function. That is, $\Psi=\left(\gamma_{1}, \gamma_{0}, \gamma_{-1}\right) \phi$, where $\gamma_{i} \geq 0$ and $\phi$ is a scalar function. Such a solution is called single mode approximation (SMA) in physics literatures. Further, the ground state preserves the population of the three components with

$$
N_{3 C}=\left(\frac{1-N_{0}+M}{2}, \frac{1-M^{2}}{2}, \frac{1-N_{0}-M}{2}\right),
$$

no matter how the strength of the coupling interactions change. (10) is obtained from the two conservation laws and minimizing the spin-exchange interaction energy. Recently, Lin and Chern [25] also theoretically proved the validity of the single-mode approximation for the spin-1 ferromagnetic BEC, and Bao et al. [36] applied this SMA ansatz to shorten the ground state computation. 
- For the antiferromagnetic system $\left(g_{s}>0\right)$, the ground state is a twocomponent $(2 \mathrm{C})$ state, where $\psi_{0} \equiv 0$. The population of each spin component always satisfies

$$
N_{2 C}=\left(\frac{1+M}{2}, 0, \frac{1-M}{2}\right) .
$$

Finally, we have concluded that in the absence of the magnetic field, the ground state is a three-component $(3 \mathrm{C})$ solution $\left(N_{j} \neq 0\right)$ if $g_{s}<0$ and is a two-component $(2 \mathrm{C})$ solution $\left(N_{0}=0\right)$ if $g_{s}>0$.

\section{The Parameter Switching Continuation Methods}

The discoveries presented in Section 4 are attributed by the continuation methods equipped with the parameter switching technique. Without this parameter switching technique, it would be non-trivial, if not impossible, to explore the new findings regarding the ground state phase diagrams of the spin-1 BECs in a magnetic field.

This particular continuation scheme is proposed on top of the pseudo-arclength continuation method [14, 29, 30, 31]. In the first stage, we employ the continuation algorithm proposed in [14] to trace the ground state solution curve by setting $q=0$ and treating both $g_{n}$ and $g_{s}$ as continuation parameters. The target point is the ground state of the spin-1 BEC in the absence of an external magnetic field. In the second stage, the solution curve starting from the target point in the previous stage is traced by treating $q$ as continuation parameter, while $g_{n}$ and $g_{s}$ are fixed. In the study of the phase transitions, we detect the bifurcation points in the second stage.

We elaborate the continuation scheme in the following sections. For convenience, the bold face letters or symbols are used to represent matrices or vectors. The approximations of wave functions $u_{j}$ and densities $n_{j}$ at grid points are denoted as $\mathbf{u}_{j}$ and $\mathbf{n}_{j}$, respectively.

\subsection{Pseudo-arclength continuation method (PACM)}

Continuation methods are reliable and powerful tools for computing multiform solutions of a system of nonlinear equations involving one or more parameters. Various algorithms based on the continuation method have been successful in solving some challenging problems [31, 34, 14, 35, 27]. Recently, some numerical methods based on the gradient flow with discrete normalization (GFDN) have been proposed for computing ground states of spin-1 BEC systems [26, 28, 36]. The GFDN mainly computes the ground states with fixed physical parameters, while the continuation methods can be used to study not only the ground state patterns but also the bifurcation diagrams on the parameter space.

By letting $\mathbf{u}=\left(\mathbf{u}_{1}^{\top}, \mathbf{u}_{0}^{\top}, \mathbf{u}_{-1}^{\top}, \mu, \lambda\right)^{\top} \in \mathbb{R}^{3 N+2}$, we write the discrete form of the CGPE (17) with the constraints (3) and (4) as the following parameterized nonlinear equation system

$$
\mathbf{G}(\mathbf{u}, \tau)=\mathbf{0}
$$


where $\mathbf{G}: \mathbb{R}^{3 N+2} \times \mathbb{R} \rightarrow \mathbb{R}^{3 N+2}$. In particular, $\tau$ is the continuation parameter depending on the problem under consideration and is incorporated into (12) by setting

$$
g_{n}=\bar{g}_{n} \tau, g_{s}=\bar{g}_{s} \tau, \text { or } q=\bar{q} \tau,
$$

where $\bar{g}_{n}, \bar{g}_{s}$ and $\bar{q}$ are the desired physical constants and $0 \leq \tau \leq 1$. By parametrizing the solution set $(\mathbf{u}, \tau)$ via arc-length in terms of $s$, we define the solution curve of (12) as

$$
\mathcal{C}=\left\{\mathbf{x}(s)=\left(\mathbf{u}(s)^{\top}, \tau(s)\right)^{\top} \mid G(\mathbf{x}(s))=\mathbf{0}, s \in \mathbb{R}\right\} .
$$

Note that the choices of the initial points of the solution curves are crucial.

We perform the PACM by the predictor-corrector procedure based on Euler's and Newton's methods. Let $\mathbf{x}_{k}=\mathbf{x}\left(s_{k}\right)=\left[\mathbf{u}^{\top}\left(s_{k}\right), \tau\left(s_{k}\right)\right]^{\top}$ be an approximating point at the $k$ th iteration. The Euler predictor is used to predict the next point

$$
\mathbf{x}_{k+1}^{(0)}=\mathbf{x}_{k}+h_{k} \dot{\mathbf{x}}_{k}
$$

where $h_{k}$ is a suitable step length and $\dot{\mathbf{x}}_{k}=\left[\left(\dot{\mathbf{u}}_{k}\right)^{\top}, \dot{\tau}_{k}\right]^{\top}$ is the unit tangent vector to the solution curve at the current point. To obtain $\dot{\mathbf{x}}_{k}$, we solve the linear system

$$
\mathcal{D} \mathbf{G}\left(\mathbf{x}_{k}\right) \dot{\mathbf{x}}_{k}=\mathbf{0}
$$

where

$$
\mathcal{D} \mathbf{G}\left(\mathbf{x}_{k}\right)=\left[\mathbf{G}_{\mathbf{u}}\left(\mathbf{x}_{k}\right), \mathbf{G}_{\tau}\left(\mathbf{x}_{k}\right)\right] \in \mathbb{R}^{(3 N+2) \times(3 N+3)}
$$

is the corresponding Jacobian matrix with respect to $s$.

Next, to obtain the correction vector, we use Newton method to solve the nonlinear system

$$
\left\{\begin{array}{l}
\mathbf{G}(\mathbf{u}, \tau)=\mathbf{0} \\
\dot{\mathbf{u}}_{k}^{\top} \mathbf{u}+\dot{\tau}_{k} \tau=\dot{\mathbf{u}}_{k}^{\top} \mathbf{u}_{k+1}^{(0)}+\dot{\tau}_{k}^{\top} \tau_{k+1}^{(0)}
\end{array}\right.
$$

with the initial guess $\mathbf{x}_{k+1}^{(0)}=\left[\mathbf{u}_{k+1}^{(0)}, \tau_{k+1}^{(0)}\right]$. We then solve the following augmented system

$$
\left[\begin{array}{cc}
G_{\mathbf{u}}\left(\mathbf{x}_{k+1}^{(i)}\right) & G_{\tau}\left(\mathbf{x}_{k+1}^{(i)}\right) \\
\dot{\mathbf{u}}_{i}^{\top} & \dot{\tau}_{k}
\end{array}\right] \delta^{(i)}=\left[\begin{array}{c}
G\left(\mathbf{x}_{k+1}^{(i)}\right) \\
\left(\mathbf{x}_{k+1}^{(i)}-\mathbf{x}_{k+1}^{(0)}\right) \cdot \dot{\mathbf{x}}_{k}
\end{array}\right]
$$

to obtain the Newton corrector

$$
\mathbf{x}_{k+1}^{(i+1)}=\mathbf{x}_{k+1}^{(i)}+\delta^{(i)}
$$

When $\mathbf{x}_{k+1}^{(i+1)}$ satisfies the convergence criterion, we let $\mathbf{x}_{k+1}=\mathbf{x}_{k+1}^{(i+1)}$ and continue next predictor-corrector step. 


\subsection{The parameter-switching technique}

By using the PACM described in Section 3.1, we can compute the ground state solutions of the spin-1 BEC with desired coupling constants in zero or nonzero magnetic field. On top of the PACM, we further propose the parameterswitching technique to find the ground state solutions of the spin- 1 BEC in the presence of the magnetic field.

In the first stage of the parameter-switching process, we compute the ground states of the spin-1 condensate in zero magnetic field under the given strength of coupling interactions (see [14] for more details). That is, we treat $g_{n}$ and $g_{s}$ as the continuation parameters and track the solution curve,

$\mathcal{C}^{(1)}=\left\{\mathbf{x}=\left(\mathbf{u}^{\top}, \tau\right)^{\top} \mid G(\mathbf{x})=\mathbf{0}\right.$ with $g_{n}=\bar{g}_{n} \tau, g_{s}=\bar{g}_{s} \tau$ and $q=0$, for $\left.0 \leq \tau \leq 1\right\}$,

which starts from the ground state solution of linear Schrödinger equation (LSE). Note that the coupling constants $g_{n}$ and $g_{s}$ can be adjusted by tuning the s-wave scattering length $a_{0}$ and $a_{2}$ via an appropriate setting of the Feshbach resonances [13, 37]. This provides the justification for the choice of the continuation parameters $g_{n}$ and $g_{s}$.

In spin-1 condensates, the ground state patterns depend on the strength of the applied magnetic field, or equivalently, the QZ parameter $q$. The parameter $q$ can be tuned experimentally via laser or microwave dressing field [15, 21, 38, 39]. Moreover, $q$ can vary from positive to negative [21, 22]. These experimental works interest us to explore the ground state patterns for both $q>0$ and $q<0$ regime numerically.

To investigate the ground state of a spin-1 BEC numerically under the influence of an external magnetic field, we further treat the QZ parameter $q$ as the continuation parameter to track the solution curve

$\mathcal{C}^{(2)}=\left\{\mathbf{x}=\left(\mathbf{u}^{\top}, \tau\right)^{\top} \mid G(\mathbf{x})=\mathbf{0}\right.$ with $g_{n}=\bar{g}_{n}, g_{s}=\bar{g}_{s}$ and $q=\bar{q} \tau$, for $\left.0 \leq \tau \leq 1\right\}$.

Note that $\mathcal{C}^{(2)}$ starts from the termination points at $\mathcal{C}^{(1)}$. The PACM used here is the same as that in the previous stage. However, the Jacobian matrix now depends on $q$ and bifurcations can occur along the solution curve. We detect the bifurcation points by monitoring the smallest eigenvalues of the augmented Jacobian matrix in continuation algorithm.

\section{Ground States and Phase Transitions}

We present the complete study of the ground states and their phase transitions for the spin-1 BECs trapped in a harmonic or box potential under the influence of the magnetic field. All the findings are obtained by using the continuation method proposed in Section 3. Table 1 summarizes all the scenarios considered in this study and the discoveries about the ground state patterns of the spin-1 BECs.

For a quick glance, the numerical results show that there are one or two bifurcation points along the ground state solution curve, depending on different 
situations (shape of the trapping potential, the sign of $g_{s}$ and the sign of $q$ ). Two types of phase transitions then occur: (i) a transition from $2 \mathrm{C}$ state to $3 \mathrm{C}$ state and vice versa (in the following, named $q_{c r 1}$ ), and (ii) a symmetry breaking of the ground state (named $q_{c r 2}$ ). The component separation is also observed for large $|q|$ and domain walls are formed.

Detailed results are presented in the following sections. In Section 4.1. Case 1 and 2 concern the antiferromagnetic condensates $\left({ }^{23} \mathrm{Na}\right)$ with box and harmonic trapping potential. In Section 4.2. Case 3 and 4 concern the ferromagnetic condensates $\left({ }^{87} \mathrm{Rb}\right)$ with box and harmonic trapping potential. In Section 4.3 . we assert the bifurcation diagrams in semi-classical regime numerically.

\subsection{Antiferromagnetic condensates}

For the antiferromagnetic system, we focus on the spin- $1{ }^{23} \mathrm{Na}$ BECs with the spin-independent interaction $g_{n}=240.8$ and the spin-exchange interaction $g_{s}=7.5$ 28]. We compute the ground states with total magnetization $M=0.3$. By using the proposed continuation scheme, the $2 \mathrm{C}$ solution satisfying (11) is first chosen as the starting point to track the solution curve (19). Then, the termination point at this curve which is the ground state of system without magnetic field is used as the starting point for the computation of curve (20).

Case 1: ${ }^{23} \boldsymbol{N a} \boldsymbol{B E C}$ with $V_{\text {ext }}(x)=0$. First, we study the case of the box potential. By increasing $q$ in the continuation algorithm, two bifurcation points $\left(q_{c r 1}=0.01759\right.$ and $\left.q_{c r 2}=0.1028\right)$ are found with rich ground state patterns. In Fig. 1, we plot the corresponding energy curves based on the reduced energy functional (8) and label these solution branches by 1, 1-1 and 1-1-1. The densities of the starting and termination states of these solution curves are plotted in Figs. 2 to 4. We highlight some observations from these figures.

- The solution curve 1 starts from the $2 \mathrm{C}$ ground state of the system in zero magnetic field. From Fig. 2, we can see that this $2 \mathrm{C}$ state is unchanged along the entire solution curve 1 . This is due to the fact that the quadratic Zeeman energy functional $\int q\left(n_{1}+n_{-1}\right) d x=q N$ is independent of the $2 \mathrm{C}$ state pattern. The populations $N_{1}$ and $N_{-1}$ can be determined from $N$ and $M$, see (111). This $2 \mathrm{C}$ state is the ground state when $q<q_{c r 1}$ and becomes an excited state when $q>q_{c r 1}$.

- The branch 1-1 is bifurcated from the solution curve 1 at $q=q_{c r 1}$. This bifurcation leads to the population transfer from $n_{1}$ and $n_{-1}$ to $n_{0}$, and $n_{-1}$ is gradually depleted. From Fig. 3, we observe that the densities of each spin component are symmetric, $n_{0}$ is immiscible with $n_{1}$ and $n_{-1}$, and two domain walls are formed. The ground states is this $3 \mathrm{C}$ (symmetric) state until $q$ meets the next bifurcation point $q_{c r 2}$.

- The branch 1-1-1 is bifurcated from the branch 1-1 at $q=q_{c r 2}$. On the branch 1-1-1, the corresponding solution is the ground state. It is asymmetric, and $n_{0}$ is immiscible with $n_{1}$ and $n_{-1}$. Eventually, in high magnetic field strength, the component $n_{-1}$ is vanished and the ground 
states become immiscible MS+NS, as shown in Fig. 4. This symmetry breaking and component separation were pointed out in [17] but with only schematic structure. Here, we compute the bifurcation point $q_{c r 2}$ precisely and present details of this phase transition process.

In contrast to the case of positive $q$, the effect of negative QZ parameter reduces the component $n_{0}$. Thus, the simulation for $q<0$ becomes trivial, since the ground state of the antiferromagnetic condensates in zero magnetic field is the $2 \mathrm{C}$ solution. The numerical results show this fact that the ground states from $q=0$ to $q=-1$ are always the same $2 \mathrm{C}$ states.

We summarize the ground state patterns and their phase diagrams of the spin- ${ }^{23} \mathrm{Na}$ BEC in the box potential with various strengths of the QZ effect in Table 2. The correspond energy and the particle number of $u_{-1}$ are also listed in the table.

Case 2: ${ }^{23} \boldsymbol{N a} \boldsymbol{B} \boldsymbol{E} \boldsymbol{C}$ with $V_{\text {ext }}(x)=\frac{1}{2} x^{2}$. We now consider the spin-1 BEC of $10^{4}{ }^{23} \mathrm{Na}$ atoms trapped in the harmonic potential. The solution curves for the case of positive $q$ are plotted in Fig. 5. The $2 \mathrm{C}$ solution curve is labelled by 1 and its solution branch by 1-1. We also depict the densities of the starting and termination states of these two curves in Figs. 6] and 7 . In addition, for the case of negative $q$, the ground states are identical $2 \mathrm{C}$ states, and no bifurcation is found. We demonstrate how the ground state structure changes with various $q$ in Table 3. We also make the following remarks for this simulation.

- From Fig. 5e observe that there is only one bifurcation $\left(q_{c r 1}=0.02606\right)$. The $3 \mathrm{C}$ APM states bifurcate from $2 \mathrm{C}$ states at $q=q_{c r 1}$. That is, this phase transition leads to the increase in $n_{0}$, but the depletion of $n_{-1}$ and $n_{1}$.

- The solutions on curve 1 are identical $2 \mathrm{C}$ states with condition (11), as shown in Fig. 6. Note that they are the ground states when $q<q_{\text {cr } 1}$. For $q>q_{c r 1}$, the $n_{0}$ state becomes positive.

- As we increase $q$, there are two situations depending on the strength $V_{0}$ of the harmonic trap potential as well as the total magnetization $M$. Before we describe the two situations, let us first explain the mechanisms of symmetry maintaining and symmetry breaking of this system. The harmonic trap potential introduces an external symmetric trapping force $-\nabla V(x)$ toward the center. This symmetric force causes a symmetric profile of $n$, which reaches minimal potential energy. On the other hand, there is an internal repulsive force between $n_{0}$ and $n_{1}\left(n_{-1}\right.$ as well). The separation of $n_{0}$ and $n_{1}\left(n_{-1}\right.$ as well) decreases the spin-exchange interaction energy and causes a symmetry breaking. The amount of this energy certainly depends on $c_{s}$ as well as $M$.

Now, we explain the two situations of the ground states as we increase $q$. The first situation occurs when $V_{0}$ is small. In this case, this symmetric external trapping force is weak and one thus observes symmetric breaking. 
If we further increase $q$, then $n_{-1}$ is almost negligible (but not zero) and $n_{1}, n_{0}$ are separated. This is the MS+NS state in the Thomas-Fermi regime.

The second situation occurs when $V_{0}$ is large. The symmetric external force is stronger than the internal repulsive force. In this case, the asymmetric profile (from the repulsive force between $n_{1}$ and $n_{0}$ ) costs higher potential energy than the symmetry one (with no phase separation at the center). Thus, the ground state profile keeps symmetric. As we further increase $q$, as shown in Table 3, the solutions on the branch 1-1 eventually become the symmetric MS+NS state in which $n_{-1}$ is almost negligible, and $n_{1}$ and $n_{0}$ are separated. These results are in good agreement with those in [17, 18, 28].

\subsection{Ferromagnetic condensates}

For the case of the ferromagnetic system, we consider the spin-1 condensate of $10^{4}{ }^{87} \mathrm{Rb}$ atoms, in which $g_{n}=885.4$ and $g_{s}=-4.1$ [28]. We first start the continuation method from the $3 \mathrm{C}$ solution with (10) to get the ground state in zero magnetic field. The obtained solution is then used as the starting point to investigate the ground state pattern under the effect of magnetic field. In contrast to the antiferromagnetic case, the ground state patterns of the ferromagnetic system are dramatically different for the negative QZ effect $q$. To our knowledge, there is no numerical study on the ferromagnetic spin- 1 in the $q<0$ regime.

Case 3: ${ }^{87} \boldsymbol{R} \boldsymbol{b} \boldsymbol{B} \boldsymbol{E} \boldsymbol{C}$ with $V_{\text {ext }}(x)=0$. First, by employing the proposed continuation algorithm along the solution curve with parameters $q=0, g_{n}=$ $885.4 \tau, g_{s}=-4.1 \tau, 0 \leq \tau \leq 1$, we obtain the ground state of ferromagnetic condensate in zero magnetic field, which is a $3 \mathrm{C}$ state. Then we take $q$ as the continuation parameter from 0 to -1 . Fig. 8 is the energy curves with $q$ ranging from 0 to -1 . Figs. 9 and 10 display the starting $(q=0)$ and target $(q=-1)$ states on these solution curves. The observations from these computations are highlighted as follows:

- In Fig. 8, the solutions on curve 1 are symmetric. Along this curve (with $q$ ranging from 0 to -1 ), the branch $1-1$ bifurcates at $q_{c r 2}=-0.01389$ which has lower energy. The solutions on the branch 1-1 are asymmetric. Thus, the ground states are symmetric for $q>q_{c r 2}$ and asymmetric for $q<q_{c r 2}$, and $q_{c r 2}$ is a symmetry-breaking bifurcation point.

- From Figs. 9 and [10, we observe that the decrease of $q$ suppresses $n_{0}$ for both symmetric and asymmetric solutions. As $q<q_{c r 2}, n_{0}$ gradually depletes to zero, $n_{1}$ and $n_{1}$ are gradually immiscible and separate. Consequently, two domain walls are formed for symmetric solutions, whereas only one domain wall is form for the asymmetric ground state. This ground state is denoted by MS+MS. 
For ferromagnetic condensates with positive $q$, we track the solution curve with $q$ ranging from 0 to 5 . The solutions start from a $3 \mathrm{C}$ state. As $q$ increases, $n_{0}$ increases, but $n_{1}$ and $n_{-1}$ decrease. Eventually, $n_{-1}$ depletes to zero and solution is a $2 \mathrm{C}$ miscible state consisting components 1 and 0 . We denote it by $2 \mathrm{C}_{\{1,0\}}$. Along this solution curve, the solutions are all symmetric and no bifurcation point is found. In Table 4 , we collect the ground state patterns with various $q$ for this simulation.

Case 4: ${ }^{87} \boldsymbol{R} \boldsymbol{b} \boldsymbol{B} \boldsymbol{E} \boldsymbol{C}$ with $V_{\text {ext }}(x)=\frac{1}{2} x^{2}$. In this simulation, we study the ground states of the spin- ${ }^{87} \mathrm{Rb}$ BEC confined in a harmonic trap. The bifurcation diagram of the ground states with negative $q$ is depicted in Fig. 11. Similar to the case of box potential, there is only one bifurcation $\left(q_{c r 2}=-0.02125\right)$ on this ground state solution curve. The $q_{c r 2}$ causes the symmetry breaking phase transition in the ground state. The properties of the ground state structure are similar to those in the box potential. We display the starting and target states of these solution curves in Figs. 12, and 13, In Table 5, we also show the ground state patterns with various $q$.

\subsection{Bifurcation diagram in semi-classical regime}

In this subsection, we further investigate the bifurcation diagrams in semiclassical regime by considering

$$
\left\{\begin{aligned}
(\mu+\lambda) u_{1} & =\left[-\epsilon_{k}^{2} \frac{\nabla^{2}}{2}+g_{n} n+q\right] u_{1}+g_{s}\left[n_{0}\left(u_{1}-\sigma u_{-1}\right)+u_{1}\left(n_{1}-n_{-1}\right)\right] \\
\mu u_{0} & =\left[-\epsilon_{k}^{2} \frac{\nabla^{2}}{2}+g_{n} n\right] u_{0}+g_{s} u_{0}\left(u_{1}-\sigma u_{-1}\right)^{2} \\
(\mu-\lambda) u_{-1} & =\left[-\epsilon_{k}^{2} \frac{\nabla^{2}}{2}+g_{n} n+q\right] u_{-1}+g_{s}\left[n_{0}\left(u_{-1}-\sigma u_{1}\right)+u_{-1}\left(n_{-1}-n_{1}\right)\right],
\end{aligned}\right.
$$

where the parameter $0<\epsilon_{k} \leq 1$ is the strength of the kinetic-energy term. We are interested in the variation of the bifurcation curves for various $\epsilon_{k}$. In our simulations, we choose $\epsilon_{k}=0.1,0.5$ and 1 . In the study below, the box potential with $V_{0}=0$ is taken into account.

In Fig. 14, we present the ground state phase diagrams of the antiferromagnetic systems $\left({ }^{23} \mathrm{Na}\right.$ with $g_{n}=240.8$ and $g_{s}=7.5$ ) with different $\epsilon_{k}$ by using the proposed continuation algorithm. The two curves in these figures indicate two bifurcation points on the ground state solution curves versus magnetization $M$ from 0.05 to 0.9 . For small $\epsilon_{k}$, the detection of the bifurcations becomes a demanding task due to the nearness of two phase transitions. We overcome this difficulty by tuning the step length used in Euler predictor (14). The proposed continuation algorithm thus enables us to determine precisely the bifurcations on the ground state solution curves. We highlight some observations found in these computations.

- In Fig. 14, we see that the two bifurcation curves $q_{c r 1}(M)$ and $q_{c r 2}(M)$ gradually merge as $\epsilon_{k}$ becomes smaller and smaller. We expect they merge as $\epsilon_{k} \rightarrow 0$. 
- It is interesting to see that the bifurcation $q_{c r 2}(M)$ bends backward for small $M$. Further, for large $\epsilon_{k}$, the region between $q_{c r 1}(M)<q<q_{c r 2}(M)$ becomes larger and larger. This means that, for small $M$, it is harder and harder to break the symmetry as we increase the strength of the applied magnetic field. This is mainly due to strong homogenization effect of the kinetic energy term.

\section{Conclusion}

Aiming at the ground state patterns and the phase diagrams of the spin-1 BECs, we have developed a numerical algorithm based on the PACM, in which the parameter-switching technique is used. By the proposed algorithm, we provide a complete investigation on the ground state patterns of spin-1 BECs over a broad range of physical parameters of interest.

For the spin-1 antiferromagnetic BEC with large enough positive QZ effect, the system undergoes two ground state phase transitions from a $2 \mathrm{C}$ state to a $3 \mathrm{C}$ symmetric state $(2 \mathrm{C} \rightarrow 3 \mathrm{C})$, then to an asymmetric phase separated state $(3 \mathrm{C} \rightarrow 2 \mathrm{C}+\mathrm{NS})$ in the case of the box trap. While only one phase transition $(2 \mathrm{C} \rightarrow 3 \mathrm{C})$ occurs in the case of harmonic traps and results in the symmetric phase separated ground state. Next, we investigate the spin-1 BEC in negative $q$ regime. Particularly, for ferromagnetic condensates, the effect of negative QZ shift leads to a symmetry breaking phase transition in both box and harmonic traps. We further study the phase transition diagrams on the $(M, q)$ plane in the semi-classical regime. It is found that the two phase transition curves $(2 \mathrm{C} \rightarrow 3 \mathrm{C}$, $3 \mathrm{C} \rightarrow 2 \mathrm{C}+\mathrm{NS}$ ) merge as the the strength of the kinetic-energy term tends to 0 . These results reveal that the proposed continuation algorithm is capable of accurately and efficiently finding all ground state patterns. Thus, a complete phase transition diagram of the spin-1 BECs in the presence of magnetic field is provided.

\section{Acknowledgements}

The authors are grateful to the anonymous referees for their useful comments and suggestions. This work is partially supported by the National Center for Theoretical Sciences and the National Science Council of the Republic of China under contract numbers: NSC 102-2115-M-134-004 (Chen), NSC 102-2115-M009-013 (Chern), and NSC 100-2628-M-002-011-MY4 (Wang).

\section{References}

[1] J. Stenger, S. Inouye, D. Stamper-Kurn, H.-J. Miesner, A. Chikkatur, W. Ketterle, Spin domains in ground-state Bose-Einstein condensates, Nature 396 (6709) (1998) 345-348. 
[2] D. Stamper-Kurn, M. Andrews, A. Chikkatur, S. Inouye, H.-J. Miesner, J. Stenger, W. Ketterle, Optical confinement of a Bose-Einstein condensate, Physical Review Letters 80 (10) (1998) 2027.

[3] T.-L. Ho, Spinor Bose condensates in optical traps, Physical review letters 81 (4) (1998) 742.

[4] T. Isoshima, K. Machida, T. Ohmi, Spin-domain formation in spinor BoseEinstein condensation, Physical Review A 60 (6) (1999) 4857.

[5] I. Bloch, J. Dalibard, W. Zwerger, Many-body physics with ultracold gases, Reviews of Modern Physics 80 (3) (2008) 885.

[6] A. Leanhardt, Y. Shin, D. Kielpinski, D. Pritchard, W. Ketterle, Coreless vortex formation in a spinor Bose-Einstein condensate, Physical review letters 90 (14) (2003) 140403.

[7] L. Sadler, J. Higbie, S. Leslie, M. Vengalattore, D. Stamper-Kurn, Spontaneous symmetry breaking in a quenched ferromagnetic spinor bose-einstein condensate, Nature 443 (7109) (2006) 312-315.

[8] Y. Kawaguchi, H. Saito, M. Ueda, Can spinor dipolar effects be observed in Bose-Einstein condensates?, Physical review letters 98 (11) (2007) 110406.

[9] H. Nistazakis, D. Frantzeskakis, P. Kevrekidis, B. Malomed, R. CarreteroGonzález, A. Bishop, Polarized states and domain walls in spinor BoseEinstein condensates, Physical Review A 76 (6) (2007) 063603.

[10] Z.-D. Li, Q.-Y. Li, P.-B. He, J.-Q. Liang, W. Liu, G. Fu, Domain-wall solutions of spinor Bose-Einstein condensates in an optical lattice, Physical Review A 81 (1) (2010) 015602.

[11] S. Hoshi, H. Saito, Symmetry-breaking magnetization dynamics of spinor dipolar Bose-Einstein condensates, Physical Review A 81 (1) (2010) 013627.

[12] B. Pasquiou, E. Maréchal, G. Bismut, P. Pedri, L. Vernac, O. Gorceix, B. Laburthe-Tolra, Spontaneous demagnetization of a dipolar spinor bose gas in an ultralow magnetic field, Physical Review Letters 106 (25) (2011) 255303.

[13] M.-S. Chang, Q. Qin, W. Zhang, L. You, M. S. Chapman, Coherent spinor dynamics in a spin-1 Bose condensate, Nature Physics 1 (2) (2005) 111-116.

[14] J.-H. Chen, I.-L. Chern, W. Wang, Exploring ground states and excited states of spin-1 Bose-Einstein condensates by continuation methods, Journal of Computational Physics 230 (6) (2011) 2222-2236.

[15] D. Jacob, L. Shao, V. Corre, T. Zibold, L. De Sarlo, E. Mimoun, J. Dalibard, F. Gerbier, Phase diagram of spin-1 antiferromagnetic Bose-Einstein condensates, Physical Review A 86 (6) (2012) 061601. 
[16] W. Zhang, S. Yi, L. You, Mean field ground state of a spin-1 condensate in a magnetic field, New Journal of Physics 5 (1) (2003) 77.

[17] M. Matuszewski, T. J. Alexander, Y. S. Kivshar, Excited spin states and phase separation in spinor Bose-Einstein condensates, Physical Review A 80 (2) (2009) 023602.

[18] M. Matuszewski, Ground states of trapped spin-1 condensates in magnetic field, Physical Review A 82 (5) (2010) 053630.

[19] M. Matuszewski, T. J. Alexander, Y. S. Kivshar, Spin-domain formation in antiferromagnetic condensates, Physical Review A 78 (2) (2008) 023632.

[20] J. Mur-Petit, Spin dynamics and structure formation in a spin-1 condensate in a magnetic field, Physical Review A 79 (6) (2009) 063603.

[21] E. M. Bookjans, A. Vinit, C. Raman, Quantum phase transition in an antiferromagnetic spinor Bose-Einstein condensate, Physical Review Letters 107 (19) (2011) 195306.

[22] A. Vinit, E. Bookjans, C. S. de Melo, C. Raman, Antiferromagnetic spatial ordering in a quenched one-dimensional spinor gas, Physical review letters 110 (16) (2013) 165301.

[23] W. Bao, Y. Zhang, Dynamical laws of the coupled gross-pitaevskii equations for spin-1 Bose-Einstein condensates, Methods and Applications of Analysis 17 (1) (2010) 49-80.

[24] D. Cao, I.-L. Chern, J.-C. Wei, On ground state of spinor Bose-Einstein condensates, NoDEA: Nonlinear Differential Equations and Applications 18 (4) (2011) 427-445.

[25] L. Lin, I.-L. Chern, A kinetic energy reduction technique and characteristics of the ground states of spin-1 Bose-Einstein condensates, Discrete and Continuous Dynamical Systems - Series B 19 (4) (2014) 1119-1128.

[26] W. Bao, F. Y. Lim, Computing ground states of spin-1 Bose-Einstein condensates by the normalized gradient flow, SIAM Journal on Scientific Computing 30 (4) (2008) 1925-1948.

[27] Y.-S. Wang, C.-S. Chien, A two-parameter continuation method for computing numerical solutions of spin-1 Bose-Einstein condensates, Journal of Computational Physics 256 (2014) 198-213.

[28] F. Y. Lim, W. Bao, Numerical methods for computing the ground state of spin-1 bose-einstein condensates in a uniform magnetic field, Physical Review E 78 (6) (2008) 066704.

[29] H. D. Mittelmann, A pseudo-arclength continuation method for nonlinear eigenvalue problems, SIAM journal on numerical analysis 23 (5) (1986) 1007-1016. 
[30] E. L. Allgower, K. Georg, Numerical continuation methods, Vol. 13, Springer-Verlag Berlin, 1990.

[31] Y.-C. Kuo, W.-W. Lin, S.-F. Shieh, W. Wang, A minimal energy tracking method for non-radially symmetric solutions of coupled nonlinear schrödinger equations, Journal of Computational Physics 228 (21) (2009) $7941-7956$.

[32] D. R. Romano, E. J. V. de Passos, Population and phase dynamics of $\mathrm{f}=1$ spinor condensates in an external magnetic field, Physical Review A 70 (4) (2004) 043614.

[33] M. Bayindir, B. Tanatar, Z. Gedik, Bose-Einstein condensation in a onedimensional interacting system due to power-law trapping potentials, Physical Review A 59 (2) (1999) 1468.

[34] C.-S. Chien, S.-L. Chang, B. Wu, Two-stage continuation algorithms for bloch waves of Bose-Einstein condensates in optical lattices, Computer Physics Communications 181 (10) (2010) 1727-1737.

[35] Y.-C. Kuo, S.-F. Shieh, W. Wang, Rotational quotient procedure: A tracking control continuation method for pdes on radially symmetric domains, Computer Physics Communications 183 (4) (2012) 998-1001.

[36] W. Bao, I.-L. Chern, Y. Zhang, Efficient numerical methods for computing ground states of spin-1 bose-einstein condensates based on their characterizations, Journal of Computational Physics 253 (2013) 189-208.

[37] E. Van Kempen, S. Kokkelmans, D. Heinzen, B. Verhaar, Interisotope determination of ultracold rubidium interactions from three high-precision experiments, Physical review letters 88 (9) (2002) 093201.

[38] F. Gerbier, A. Widera, S. Fölling, O. Mandel, I. Bloch, Resonant control of spin dynamics in ultracold quantum gases by microwave dressing, Physical Review A 73 (4) (2006) 041602.

[39] S. Leslie, J. Guzman, M. Vengalattore, J. D. Sau, M. L. Cohen, D. StamperKurn, Amplification of fluctuations in a spinor Bose-Einstein condensate, Physical Review A 79 (4) (2009) 043631.

[40] B. Seaman, L. Carr, M. Holland, Effect of a potential step or impurity on the Bose-Einstein condensate mean field, Physical Review A 71 (3) (2005) 033609.

[41] K. Kasamatsu, M. Tsubota, Multiple domain formation induced by modulation instability in two-component Bose-Einstein condensates, Physical review letters 93 (10) (2004) 100402.

[42] M. Moreno-Cardoner, J. Mur-Petit, M. Guilleumas, A. Polls, A. Sanpera, M. Lewenstein, Predicting spinor condensate dynamics from simple principles, Physical review letters 99 (2) (2007) 020404. 
[43] M. Yasunaga, M. Tsubota, Ferromagnetic resonance in spinor dipolar BoseEinstein condensates, Physical Review A 83 (1) (2011) 013618. 
Table 1: Summaries of the ground state patterns of the spin-1 BECs.

\begin{tabular}{|c|c|c|c|}
\hline $\mathrm{BEC}$ & Potential & QZ effect & Ground state patterns \\
\hline \multirow{2}{*}{${ }^{23} \mathrm{Na}$} & Constant (Case 1) & $\begin{array}{l}q>0^{*} \\
q=0 \\
q<0^{\dagger}\end{array}$ & $\begin{array}{l}2 \mathrm{C} \rightarrow 3 \mathrm{C} \rightarrow 2 \mathrm{C}+\mathrm{NS} \rightarrow \mathrm{MS}+\mathrm{NS} \\
2 \mathrm{C} \\
2 \mathrm{C}\end{array}$ \\
\hline & Harmonic (Case 2) & $\begin{array}{l}q>0^{\ddagger} \\
q=0 \\
q<0^{\dagger}\end{array}$ & $\begin{array}{l}2 \mathrm{C} \rightarrow 3 \mathrm{C} \rightarrow 2 \mathrm{C}+\mathrm{NS} \rightarrow \mathrm{MS}+\mathrm{NS} \\
2 \mathrm{C} \\
2 \mathrm{C}\end{array}$ \\
\hline \multirow{2}{*}{${ }^{87} \mathrm{Rb}$} & Constant (Case 3) & $\begin{array}{l}q>0^{*} \\
q=0 \\
q<0^{\dagger}\end{array}$ & $\begin{array}{l}3 \mathrm{C} \rightarrow 2 \mathrm{C}_{\{1,0\}} \\
3 \mathrm{C} \\
3 \mathrm{C} \rightarrow 2 \mathrm{C}+\mathrm{NS} \rightarrow \mathrm{MS}+\mathrm{MS}+\mathrm{NS} \rightarrow \mathrm{MS}+\mathrm{MS}\end{array}$ \\
\hline & Harmonic (Case 4) & $\begin{array}{l}q>0^{\ddagger} \\
q=0 \\
q<0^{\dagger}\end{array}$ & $\begin{array}{l}3 \mathrm{C} \rightarrow 2 \mathrm{C}_{\{1,0\}} \\
3 \mathrm{C} \\
3 \mathrm{C} \rightarrow 2 \mathrm{C}+\mathrm{NS} \rightarrow \mathrm{MS}+\mathrm{MS}+\mathrm{NS} \rightarrow \mathrm{MS}+\mathrm{MS}\end{array}$ \\
\hline
\end{tabular}

* Only schematic drawings in literatures [17 and never been numerically studied.

$\dagger$ Only been observed in experiments [21, 22] and never been numerically studied.

$\ddagger$ Agree with literatures [17, 18, 28].

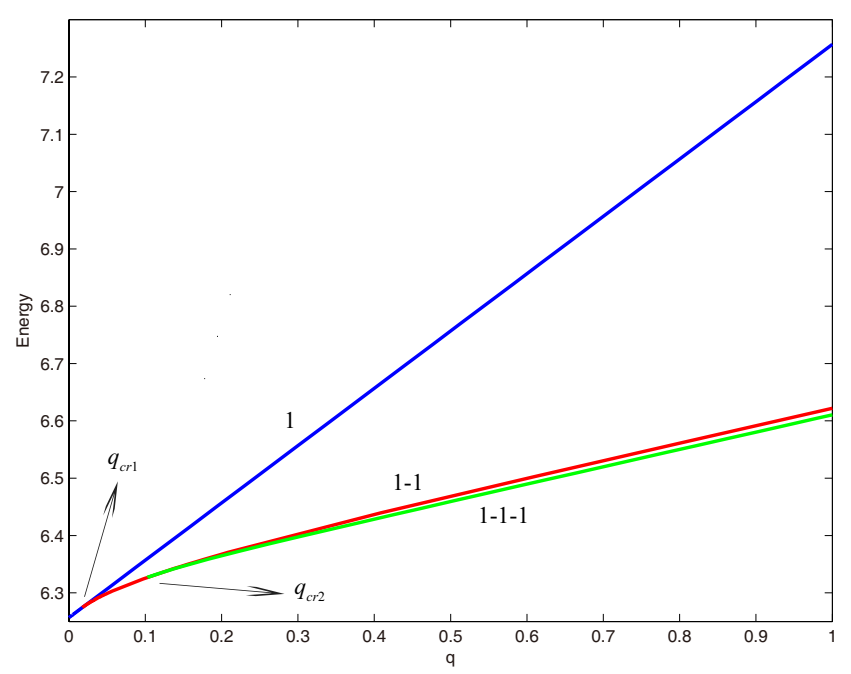

Figure 1: (Case 1) Energy curves of ${ }^{23} \mathrm{Na}$ with $M=0.3$ in the box potential. There are two bifurcation points $q_{c r 1}=0.01759$ and $q_{c r 2}=0.1028$. 

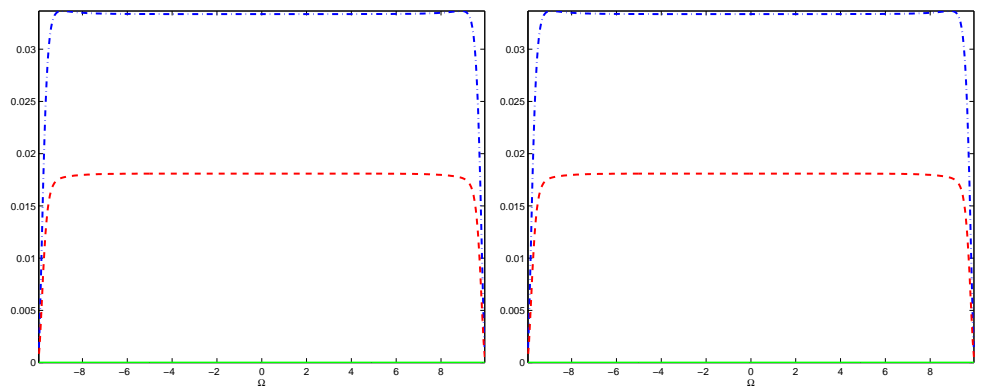

Figure 2: (Case 1) Densities of the starting state $q=0$ (left) and the target state $q=1$ (right) of curve 1 in Fig. 1 The $m_{F}=1,0,-1$ components are depicted by blue dash-dotted, green solid and red dashed lines, respectively.
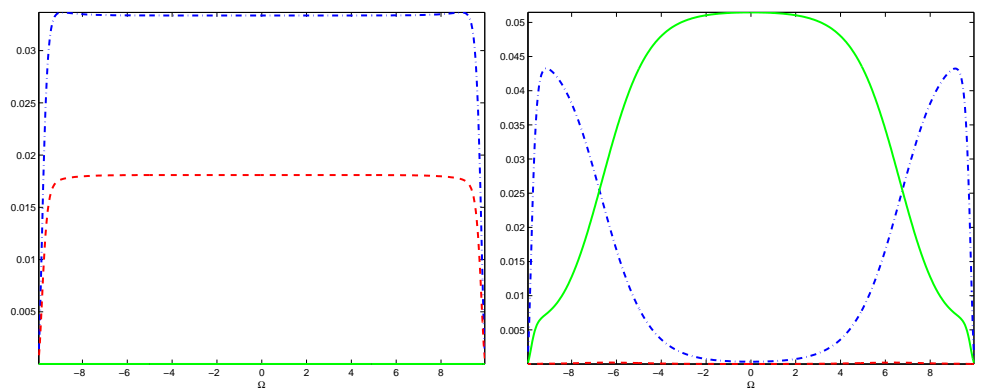

Figure 3: (Case 1) Densities of the starting state $q=0$ (left) and the target state $q=1$ (right) of curve 1-1 in Fig. 1 The $m_{F}=1,0,-1$ components are depicted by blue dash-dotted, green solid and red dashed lines, respectively.
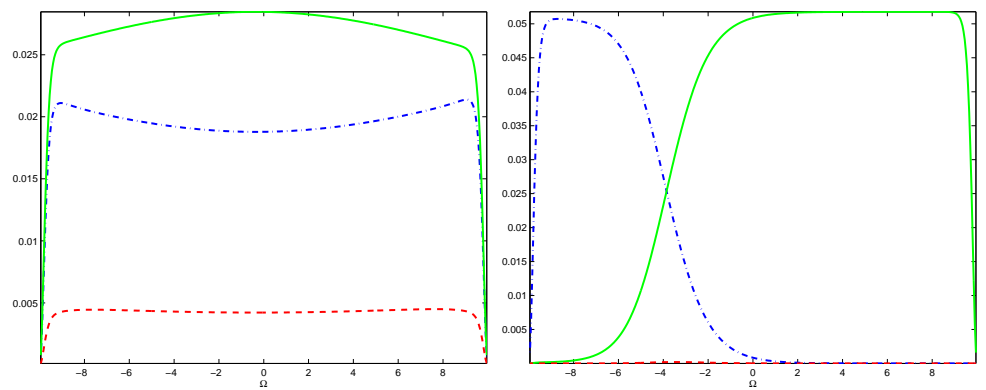

Figure 4: (Case 1) Densities of the starting state $q=0$ (left) and the target state $q=1$ (right) of curve 1-1-1 in Fig. 1 The $m_{F}=1,0,-1$ components are depicted by blue dash-dotted, green solid and red dashed lines, respectively. 
Table 2: (Case 1) Ground state patterns of antiferromagnetic BEC $\left({ }^{23} \mathrm{Na}\right)$ with $M=0.3$ in the box potential.

\begin{tabular}{ccccccc}
\hline & $q=-1$ & $q=0$ & $q=0.02582$ & $q=0.1115$ & $q=1$ & $q=3$ \\
\hline Energy $(\tilde{\mathcal{E}})$ & 5.2568 & 6.2568 & 6.2818 & 6.3309 & 6.6105 & 7.2114 \\
$N_{-1}$ & 0.35 & 0.35 & 0.2673 & 0.07373 & 0.0007 & 0.00008 \\
State & $2 \mathrm{C}$ & $2 \mathrm{C}$ & $3 \mathrm{C}$ & $2 \mathrm{C}+\mathrm{NS}$ & $2 \mathrm{C}+\mathrm{NS}$ & $\mathrm{MS}+\mathrm{NS}$ \\
& & & & & & \\
Profile & & & & & & \\
\hline
\end{tabular}

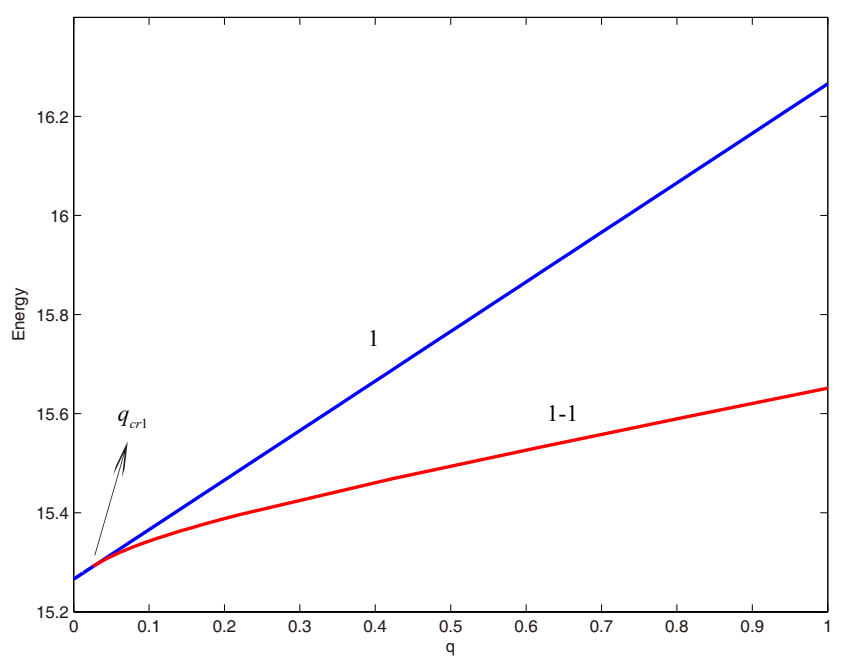

Figure 5: (Case 2) Energy curves of ${ }^{23} \mathrm{Na}$ with $M=0.3$ in the harmonic potential. There is one bifurcation point $q_{c r 1}=0.02606$.
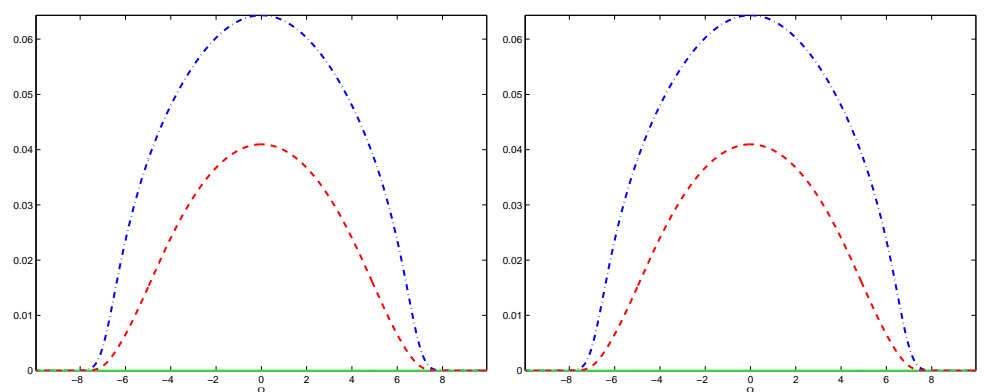

Figure 6: (Case 2) Densities of the starting state $q=0$ (left) and the target state $q=1$ (right) of curve 1 in Fig. 5 The $m_{F}=1,0,-1$ components are depicted by blue dash-dotted, green solid and red dashed lines, respectively. 

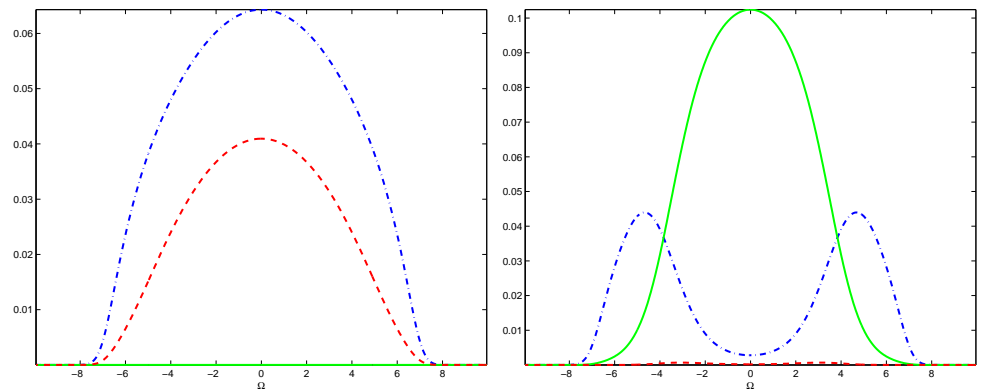

Figure 7: (Case 2) Densities of the starting state $q=0$ (left) and the target state $q=1$ (right) of curve 1-1 in Fig. 5 The $m_{F}=1,0,-1$ components are depicted by blue dash-dotted, green solid and red dashed lines, respectively.

Table 3: (Case 2) Ground state patterns of antiferromagnetic BEC $\left({ }^{23} \mathrm{Na}\right)$ with $M=0.3$ in the harmonic potential.

\begin{tabular}{|c|c|c|c|c|c|}
\hline & $q=-1$ & $q=0$ & $q=0.1537$ & $q=1$ & $q=8$ \\
\hline Energy $(\tilde{\mathcal{E}})$ & 14.2659 & 15.2659 & 15.3686 & 15.6515 & 17.7593 \\
\hline$N_{-1}$ & 0.35 & 0.35 & 0.0724 & 0.004 & 0.00008 \\
\hline State & $2 \mathrm{C}$ & $2 \mathrm{C}$ & $2 \mathrm{C}+\mathrm{NS}$ & $2 \mathrm{C}+\mathrm{NS}$ & $\mathrm{MS}+\mathrm{NS}$ \\
\hline
\end{tabular}

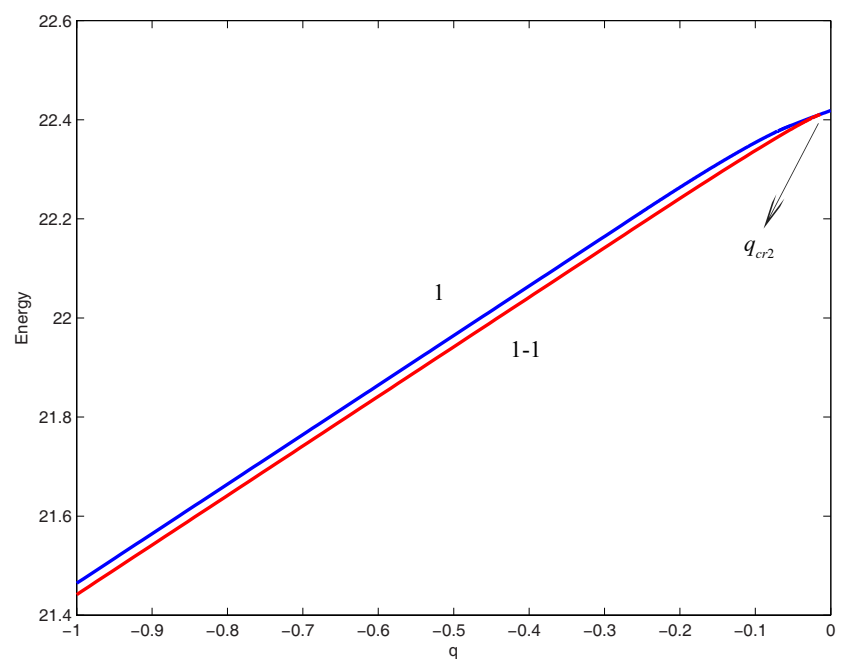

Figure 8: (Case 3) Energy curves of ${ }^{87} \mathrm{Rb}$ with $M=0.3$ in the box potential. There is one bifurcation point $q_{c r 2}=-0.01389$. 

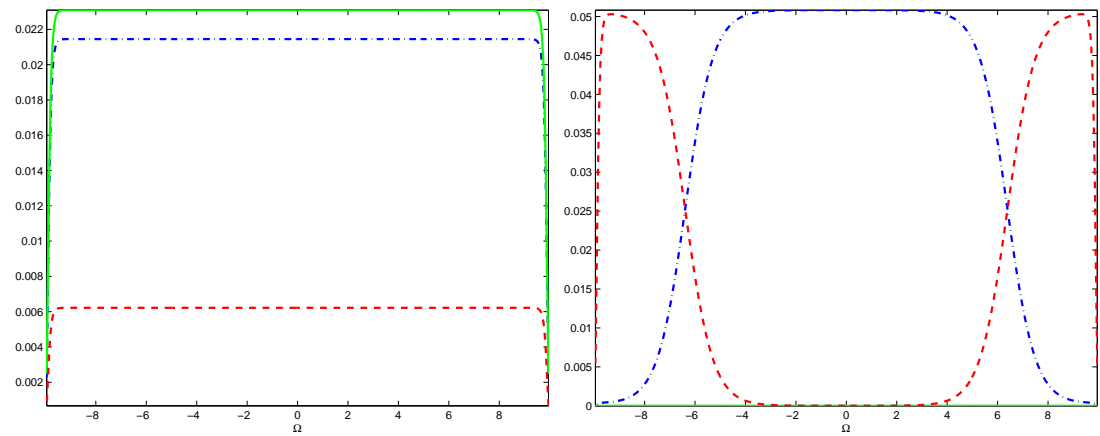

Figure 9: (Case 3) Densities of the starting state $q=0$ (left) and the target state $q=-1$ (right) of curve 1 in Fig. 8 The $m_{F}=1,0,-1$ components are depicted by blue dash-dotted, green solid and red dashed lines, respectively.
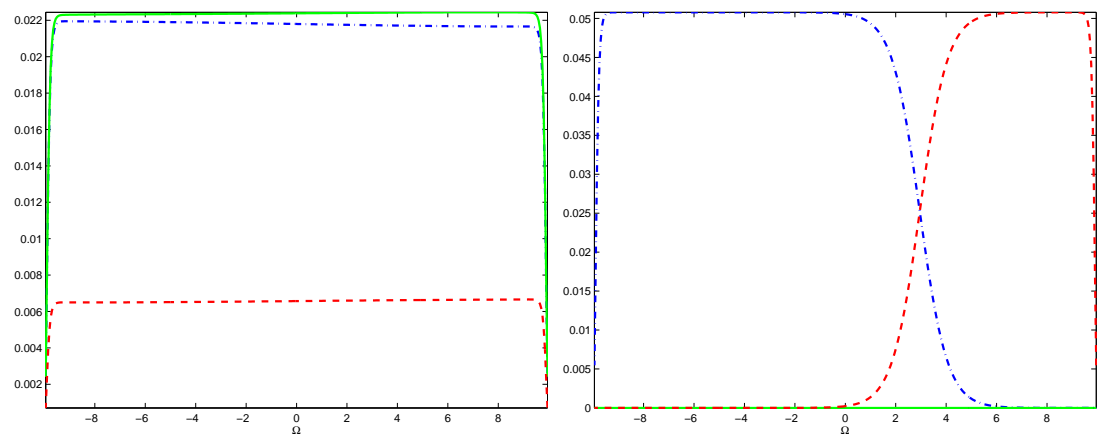

Figure 10: (Case 3) Densities of the starting state $q=0$ (left) and the target state $q=-1$ (right) of curve 1-1 in Fig. 8 The $m_{F}=1,0,-1$ components are depicted by blue dash-dotted, green solid and red dashed lines, respectively.

Table 4: (Case 3) Ground state patterns of ferromagnetic BEC $\left({ }^{87} \mathrm{Rb}\right)$ with $M=0.3$ in the box potential.

\begin{tabular}{ccccccc}
\hline & $q=-1$ & $q=-0.2035$ & $q=-0.01416$ & $q=0$ & $q=1$ & $q=5$ \\
\hline Energy $(\tilde{\mathcal{E}})$ & 21.4417 & 22.2379 & 22.4109 & 22.4187 & 22.7659 & 23.9687 \\
$N_{-1}$ & 0.35 & 0.3438 & 0.1337 & 0.1225 & 0.0018 & 0.00007 \\
State & $\mathrm{MS}+\mathrm{MS}$ & $\mathrm{MS}+\mathrm{MS}+\mathrm{NS}$ & $2 \mathrm{C}+\mathrm{NS}$ & $3 \mathrm{C}$ & $3 \mathrm{C}$ & $2 \mathrm{C}_{\{1,0\}}$ \\
& & &
\end{tabular}




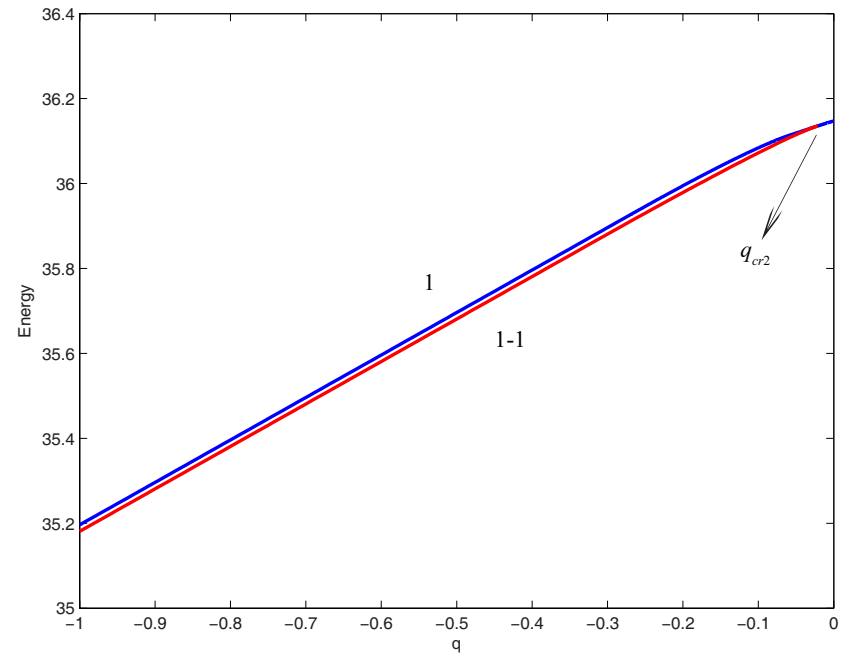

Figure 11: (Case 4) Energy curves of ${ }^{87} \mathrm{Rb}$ with $M=0.3$ in the harmonic potential. There is one bifurcation point $q_{c r 2}=-0.02125$.
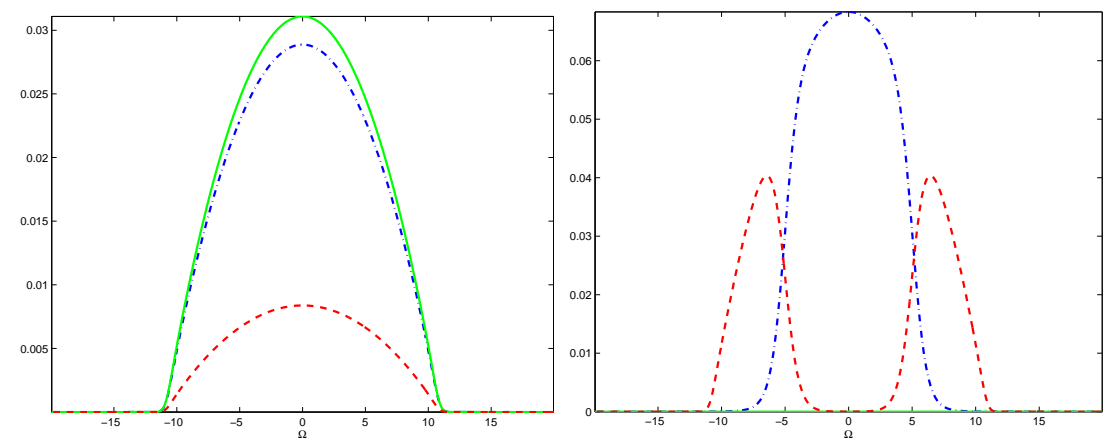

Figure 12: (Case 4) Densities of the starting state $q=0$ (left) and the target state $q=-1$ (right) of curve 1 in Fig. 11 The $m_{F}=1,0,-1$ components are depicted by blue dash-dotted, green solid and red dashed lines, respectively. 

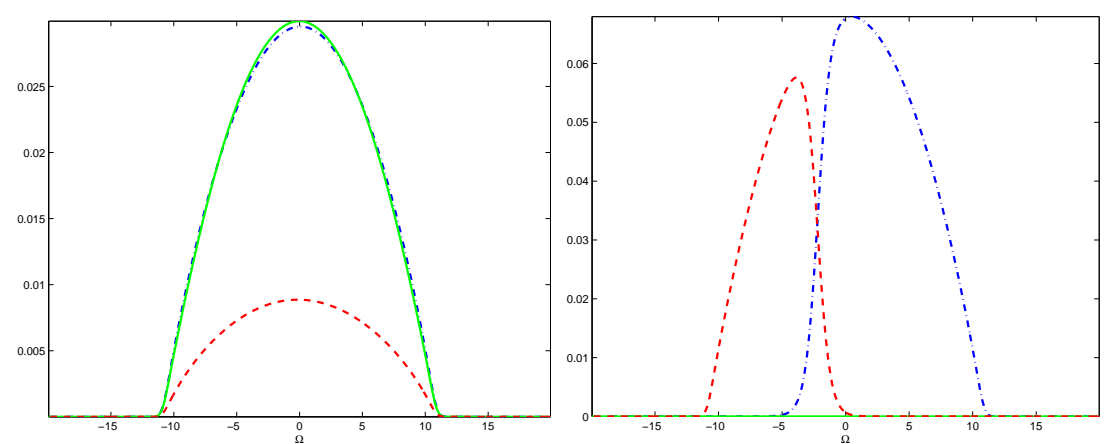

Figure 13: (Case 4) Densities of the starting state $q=0$ (left) and the target state $q=-1$ (right) of curve 1-1 in Fig. 11] The $m_{F}=1,0,-1$ components are depicted by blue dashdotted, green solid and red dashed lines, respectively.

Table 5: (Case 4) Ground state patterns of ferromagnetic BEC $\left({ }^{87} \mathrm{Rb}\right)$ with $M=0.3$ in the harmonic potential.

\begin{tabular}{ccccccc}
\hline & $q=-1$ & $q=-0.06099$ & $q=-0.02195$ & $q=0$ & $q=1$ & $q=5$ \\
\hline Energy $(\tilde{\mathcal{E}})$ & 35.1809 & 36.1352 & 36.10605 & 36.1474 & 36.4957 & 37.6991 \\
$N_{-1}$ & 0.35 & 0.2658 & 0.1399 & 0.1225 & 0.0021 & 0.00008 \\
State & MS+MS & MS+MS+NS & $2 \mathrm{C}+\mathrm{NS}$ & $3 \mathrm{C}$ & $3 \mathrm{C}$ & $2 \mathrm{C}_{\{1,0\}}$ \\
& & & &
\end{tabular}



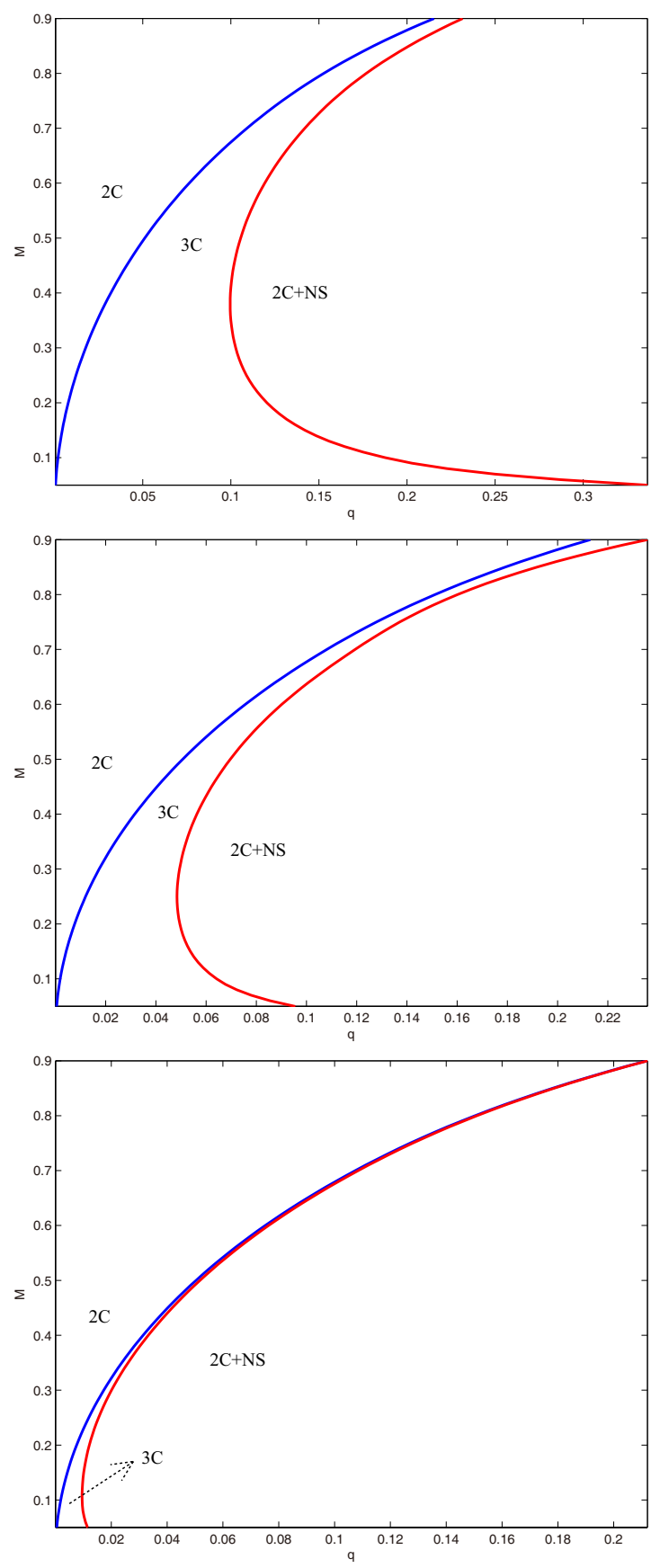

Figure 14: (semi-classical regime) The ground state phase diagram of the spin-1 ${ }^{23} \mathrm{Na}$ REC with $\epsilon_{k}=1, \epsilon_{k}=0.5$, and $\epsilon_{k}=0.1$ (from top to bottom).

26 